Homology, Homotopy and Applications, vol.8(1), 2006, pp.243-256

\title{
FAMILIES OF HOPF ALGEBRAS OF TREES AND PRE-LIE ALGEBRAS
}

\author{
PEPIJN VAN DER LAAN AND IEKE MOERDIJK
}

(communicated by U. Rehmann)

\begin{abstract}
We study Hopf algebra structures on polynomial algebras generated by coloured trees. In particular, we establish the existence of a $2 n$-parameter family of such structures for trees coloured by $n$ colours.
\end{abstract}

\section{Introduction}

Over the past years, more and more examples of combinatorial Hopf algebras appeared in the mathematical literature (cf. Kreimer [7], Connes-Kreimer [3], Loday-Ronco [8], Brouder-Frabetti [1]). In most cases these Hopf algebras are constructed one at a time. One of the authors [10] constructs such Hopf algebras in families, by freely adjoining unary operations to Hopf operads, rather than as isolated examples. For example, the Connes-Kreimer Hopf algebra of rooted trees and its planar analogue (cf. Foissy [4] for a detailed account) are examples related by a change of operad (see [10]). The non-planar version corresponds to a particular coproduct based on the commutative operad, whereas the planar version corresponds to its analogue for the associative operad. In [11], a construction along these lines is given of the Hopf algebras in $[\mathbf{8}]$ and $[\mathbf{1}]$, this time by freely adjoining a binary operation to a suitable operad.

The purpose of this paper is to extend the methods of [10], and prove the existence of $2 n$-parameter families of Hopf algebra structures on the symmetric algebra generated by rooted trees with edges coloured by $n$ colours. We start in Section 2 with the definition of the initial pair $\left(C_{n}, \lambda_{n}\right)$, consisting of a commutative algebra $C_{n}$ and a linear map $\lambda_{n}: C_{n} \otimes \cdots \otimes C_{n} \longrightarrow C_{n}$ defined on the $n$-fold tensor product. We show that from the universal property of the pair $\left(C_{n}, \lambda_{n}\right)$ alone, it follows that there exists a family of Hopf algebra structures on $C_{n}$. In Section 3, we identify $C_{n}$ with the symmetric algebra on rooted trees with $n$-coloured edges. Consequently, the Hopf algebras constructed in Section 2 are bialgebras of trees. In Section 4, we then derive an explicit formula for the corresponding coproducts in terms of trees. In Section 5, we give a description of the Lie algebra of primitive elements of the dual Hopf algebra and we give a criterion for when this is the associated Lie algebra of a pre-Lie algebra. Section 6 interprets the family of coproducts in terms of deformation theory. Finally, Section 7 sketches the more general framework of Hopf

Received February 21, 2005; published on December 22, 2005.

2000 Mathematics Subject Classification: 18D50, 16W30.

Key words and phrases: Hopf algebras, trees, deformations.

Copyright (c) 2005, International Press. Permission to copy for private use granted. 
operads and lists the results obtained when one starts from associative instead of commutative algebras.

\section{Hopf algebra structures from initial objects}

For definiteness, we work in the category of vector spaces over a field $k$ of characteristic zero. (However, with the exception of Sections 5 and 6, our arguments apply in a much more general context of modules over an algebra in any symmetric monoidal additive category.) By algebra we will always mean associative algebra with unit.

Definition 2.1. A commutative $n$-algebra is a pair $(A, \alpha)$, consisting of a commutative algebra $A$ and a linear map $\alpha: A^{\otimes n} \longrightarrow A$. A morphism of $n$-algebras $f:(A, \alpha) \longrightarrow(B, \beta)$ is an algebra homomorphism $f: A \longrightarrow B$ such that $\beta \circ f^{\otimes n}=$ $f \circ \alpha$.

We will write $\mathcal{C}_{n}$ for the category of these $n$-algebras. For general reasons, this category $\mathcal{C}_{n}$ has an initial object, the free $n$-algebra on the empty set of generators. This initial algebra will be denoted by $\left(C_{n}, \lambda_{n}\right)$. It is completely characterised (up to isomorphism) by the property that any $n$-algebra $(A, \alpha)$ admits a unique morphism $\left(C_{n}, \lambda_{n}\right) \longrightarrow(A, \alpha)$. We will give an explicit description of this initial algebra in Proposition 3.2 below.

In this section, we will show that $C_{n}$ carries a family of bialgebra (in fact Hopf algebra) structures, derived from its universal property alone. First, we need a notation. If $(A, \alpha) \in \mathcal{C}_{n}$ is a commutative $n$-algebra and $\sigma_{1}, \sigma_{2}: A^{\otimes n} \longrightarrow A$ are two linear maps, $A^{\otimes n}$ acquires the structure of a $n$-algebra by the linear map $\left(\sigma_{1}, \sigma_{2}\right):(A \otimes A)^{\otimes n} \longrightarrow A \otimes A$, defined as

$$
\left(\sigma_{1}, \sigma_{2}\right)=\left(\sigma_{1} \otimes \alpha+\alpha \otimes \sigma_{2}\right) \circ \tau .
$$

(Here $\tau:\left(A^{\otimes 2}\right)^{\otimes n} \longrightarrow A^{\otimes n} \otimes A^{\otimes n}$ is the shuffle isomorphism that separates the first and second tensor factors from $A^{\otimes 2}$.)

We are going to use this in the context where $(A, \alpha)$ is the initial $n$-algebra $\left(C_{n}, \lambda_{n}\right)$. First note that $\left(C_{n}, \lambda_{n}\right)$ is an augmented algebra. Indeed, the ground field $k$ is naturally an $n$-algebra when equipped with the zero map $k^{\otimes n} \longrightarrow k$. So by initiality of $\left(C_{n}, \lambda_{n}\right)$, there is a unique morphism of $n$-algebras $\varepsilon:\left(C_{n}, \lambda\right) \longrightarrow(k, 0)$.

Also by initiality, for any pair of $n$-ary linear maps $\sigma_{1}, \sigma_{2}: C_{n}^{\otimes n} \longrightarrow C_{n}$ as above, there is a unique morphism $\Delta:\left(C_{n}, \lambda_{n}\right) \longrightarrow\left(C_{n} \otimes C_{n},\left(\sigma_{1}, \sigma_{2}\right)\right)$ in $\mathcal{C}_{n}$. That is, a unique algebra morphism $\Delta$ such that the diagram

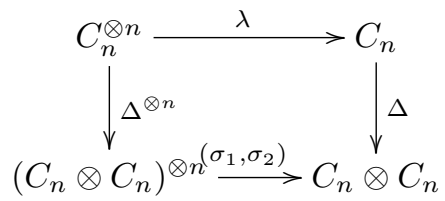

commutes. Exactly as in the case where $n=1$ treated in [10], one can prove the following Theorem. 
Theorem 2.2. Let $\Delta: C_{n} \longrightarrow C_{n} \otimes C_{n}$ be the diagonal associated to two linear maps $\sigma_{1}$ and $\sigma_{2}$. If both $\sigma_{i}$ satisfy

$$
\varepsilon \circ \sigma_{i}=\varepsilon^{\otimes n}
$$

and

$$
\Delta \circ \sigma_{i}=\left(\sigma_{i} \otimes \sigma_{i}\right) \circ \tau \circ \Delta^{\otimes n} ;
$$

then this diagonal is coassociative, and provides $C_{n}$ with the structure of a bialgebra.

Remark 2.3. We will see in the next section that the initial algebra $C_{n}$ has a natural grading. The coalgebra structure given by a pair of maps $\sigma_{1}$ and $\sigma_{2}$ will respect this grading if these maps $\sigma_{1}$ and $\sigma_{2}$ do, and consequently there exists an antipode for the bialgebra structure. A special case of this occurs in Theorems 4.1 and 4.3 below.

Remark 2.4. The algebras $C_{n}$ together form a simplicial algebra. The simplicial operations $d_{i}: C_{n} \longrightarrow C_{n-1}$ and $s_{i}: C_{n} \longrightarrow C_{n+1}$ are the algebra homomorphisms determined by

$$
\begin{array}{rlrl}
d_{0}\left(\lambda_{n}\right)\left(x_{1}, \ldots, x_{n}\right) & =\mu\left(x_{1}, \lambda_{n-1}\left(x_{2}, \ldots, x_{n}\right)\right) & \\
d_{i}\left(\lambda_{n}\right)\left(x_{1}, \ldots, x_{n}\right) & =\lambda_{n}\left(x_{1}, \ldots, \mu\left(x_{i}, x_{i+1}\right), \ldots, x_{n}\right) & & (i=1, \ldots, n-1) \\
d_{n}\left(\lambda_{n}\right)\left(x_{1}, \ldots, x_{n}\right) & =\mu\left(\lambda\left(x_{1}, \ldots, x_{n-1}\right), x_{n}\right) & \\
s_{i}\left(\lambda_{n}\right)\left(x_{1}, \ldots, x_{n}\right) & =\lambda_{n+1}\left(x_{1}, \ldots, x_{i}, 1, x_{i+1}, \ldots, x_{n}\right) & & (i=0, \ldots, n),
\end{array}
$$

similar to the formulas for the Hochschild complex. We do not know the significance of this observation.

\section{Initial algebras and trees}

Definition 3.1. A rooted tree is an isomorphism class $t$ of finite partially ordered sets which

(i) have a minimal element $r(\forall u \neq r: r<u)$, called the root, and

(ii) satisfy the tree condition that $(v \neq w) \wedge(v<u) \wedge(w<u)$ implies $(v<w) \vee(w<v)$.

In general, we will not be very precise in distinguishing between an isomorphism class $t$ and any of the posets which represent it; in particular, we will often use $t$ to denote a representing poset, and refer to it as a tree.

The elements of a tree are called vertices. A pair of vertices $v<w$ is called an edge if there is no vertex $x$ such that $v<x<w$. The number of vertices of a tree $t$ is denoted by $|t|$. A path from $w$ to $v$ in a tree is a sequence $\left(v_{i}\right)_{i}$ of elements $w=v_{n}>v_{n-1}>\cdots>v_{1}>v_{0}=v$ of maximal length. We will say that $w$ is above $v$ in a tree if there is a path from $w$ to $v$.

A forest is a finite (possibly empty) multiset (i.e., a set with multiplicities) of trees. A subforest $s$ of a rooted tree or forest $f$ is a subset of the multiset (representing) $f$ with the induced partial ordering. For a forest $f$ and a subforest $g \subset f$, we will write $g^{c}$ for the complement (as a multiset) of $g$ in $f$. Thus $g^{c}$ is a again a forest (even if $f$ and $g$ are trees, $g^{c}$ need not be a tree). 
In the sequel, we need trees with coloured edges. These are isomorphism classes of posets as above, equipped with a function from the set of edges to a fixed set of colours. The isomorphisms are required to respect the colours. In particular, an $n$-coloured tree is such a tree whose edges are coloured by the set $\{1, \ldots, n\}$ of colours. We will write $T_{n}$ for the vector space spanned by the set of such $n$-coloured trees, and $S\left(T_{n}\right)$ for the symmetric algebra on $T_{n}$.

Proposition 3.2. There is a natural algebra isomorphism between the initial $n$-algebra $C_{n}$ and the symmetric algebra $S\left(T_{n}\right)$ on the set of $n$-coloured trees.

Proof. The symmetric algebra $S\left(T_{n}\right)$ can be identified with the vector space spanned by the set of $n$-coloured forests, with the unit represented by the empty forest and the product by the disjoint union of forests. There is an operation

$$
\lambda: S\left(T_{n}\right)^{\otimes n} \longrightarrow S\left(T_{n}\right)
$$

which takes an $n$-tuple of $n$-coloured forests $f_{1}, \ldots, f_{n}$, and combines them into a single $n$-coloured tree by adding a new root, while connecting this new root to each of the roots in the forest $f_{i}$ by an edge of colour $i$.

This operation makes $S\left(T_{n}\right)$ into an object of the category $\mathcal{C}_{n}$. Since initial objects are unique up to isomorphism in any category, it now suffices to prove that $S\left(T_{n}\right)$ is initial in $\mathcal{C}_{n}$.

To this end, let $(A, \alpha)$ be any object of $\mathcal{C}_{n}$, where $\alpha: A^{\otimes n} \longrightarrow A$. Define a morphism

$$
\varphi:\left(S\left(T_{n}\right), \lambda\right) \longrightarrow(A, \alpha)
$$

by induction on trees and forests. If $f=t_{1} \cdots t_{k}$ is a forest consisting of $k$ trees, then $\varphi(f)=\varphi\left(t_{1}\right) \cdots \cdots\left(t_{k}\right)$, so it suffices to define $\varphi$ on trees. If $t$ is a tree consisting of a root only, then $\varphi(t)=\alpha(1, \ldots, 1)$. If $t$ consists of a root $r$ onto which an $n$-tuple of $n$-coloured forests $f_{1}, \ldots, f_{n}$ is attached by joining the root of each tree in $f_{i}$ to $r$ via an edge of colour $i$, then $\varphi(t)=\alpha\left(\varphi\left(f_{1}\right), \ldots, \varphi\left(f_{n}\right)\right)$. It is straightforward to check that $\varphi:\left(S\left(T_{n}\right), \lambda\right) \longrightarrow(A, \alpha)$ thus defined is indeed a morphism in $\mathcal{C}_{n}$, and is the unique such.

\section{Hopf algebras of trees}

In this section, we study a particular example of a family of Hopf algebras which can be obtained by the general method of Theorem 2.2.

Here and in the sequel $|f|$ denotes the number of vertices in the forest corresponding to $f \in S\left(T_{n}\right)$, while the associative multiplication on $S\left(T_{n}\right)$ is denoted by $\cdot$. Below, we write $\Delta\left(f_{i}\right)=\sum f_{i}^{\prime} \otimes f_{i}^{\prime \prime}$ reminiscent of the form $\Delta$ takes in a basis.

Theorem 4.1. The symmetric algebra $S\left(T_{n}\right)$ on $n$-coloured trees has a natural family of graded connected Hopf algebra structures, indexed by sequences

$$
\left(q_{11}, \ldots, q_{1 n}, q_{21}, \ldots, q_{2 n}\right) \in k^{2 n} .
$$


The grading is with respect to the number of vertices of the trees. An inductive description of the coproduct is given by

$$
\begin{aligned}
\Delta\left(\lambda\left(f_{1}, \ldots, f_{n}\right)\right)= & \sum q_{11}^{\left|f_{1}^{\prime}\right|} \cdots \cdots q_{1 n}^{\left|f_{n}^{\prime}\right|} \cdot f_{1}^{\prime} \cdots \cdots f_{n}^{\prime} \otimes \lambda\left(f_{1}^{\prime \prime}, \ldots, f_{n}^{\prime \prime}\right) \\
& +\sum \lambda\left(f_{1}^{\prime}, \ldots, f_{n}^{\prime}\right) \otimes q_{21}^{\left|f_{1}^{\prime \prime}\right|} \cdots \cdots q_{2 n}^{\left|f_{n}^{\prime \prime}\right|} \cdot f_{1}^{\prime \prime} \cdots \cdots f_{n}^{\prime \prime},
\end{aligned}
$$

where $\lambda\left(f_{1}, \ldots, f_{n}\right)$ is the rooted tree obtained for $n$ forests $f_{1}, \ldots, f_{n}$ by adding a new root and connecting each of the roots of trees in $f_{i}$ to the new root by an edge of colour $i$, and where $\left|f_{i}\right|$ is the number of vertices in the forest $f_{i}$.

Proof. We use the identification of the symmetric algebra $S\left(T_{n}\right)$ with the initial algebra $C_{n}$ given by Proposition 3.2. For a given sequence $q_{11}, \ldots, q_{1 n}, q_{21}, \ldots, q_{2 n}$ consider the maps $\sigma_{i}: S(T-n) \otimes \cdots \otimes S\left(T_{n}\right) \longrightarrow S\left(T_{n}\right)$ defined for $f_{i} \in S\left(T_{n}\right)$ by

$$
\sigma_{i}\left(f_{1}, \ldots f_{n}\right)=\left(\prod_{j} q_{i j}^{\left|f_{j}\right|}\right) \cdot f_{1} \cdots f_{n} .
$$

These maps are readily seen to satisfy the conditions of Theorem 2.2 . Thus there is a unique bialgebra structure given by a diagonal for which the diagram 1 commutes. The inductive description in the theorem is another way to express commutativity. Next, the bialgebra $S\left(T_{n}\right)$ is graded connected with respect to the grading |.|. It is well known (cf. Milnor and Moore [9]) that any graded connected bialgebra admits an antipode.

We now turn to the question of finding a more direct description of these Hopf algebra structures. For a tree $t$, a subforest $g \subset t$, and a vertex $v \in g$, we denote by $p_{k}(v, g, t)$ the number of edges of colour $k$ in the path in $t$ from $v$ to the root of $t$ that have their lower vertex in $g^{c}$. For forests $f$, we define $p_{k}(v, g, f)$ as $p_{k}(v, g \cap t, t)$, where $t$ is the connected component of $f$ containing $v$. There is an easy but useful lemma on the calculus of the $p_{k}$.

Lemma 4.2. Let $g$ and $h$ be subforests of a forest $f$. Let $v \in h$ and set $g^{\prime}=g \cup v$, $h^{\prime}=h \cap g^{\prime}, g^{\prime \prime}=g^{c} \cup v$ and $h^{\prime \prime}=h \cap g^{\prime \prime}$. Then

$$
p_{k}(v, h, f)=p_{k}\left(v, h^{\prime}, g^{\prime}\right)+p_{k}\left(v, h^{\prime \prime}, g^{\prime \prime}\right),
$$

where $g^{\prime}, g^{\prime \prime}, h^{\prime}$ and $h^{\prime \prime}$ are interpreted as subforests of $f$.

Proof. The lemma follows at once when we observe that a vertex in the path from $v$ to the root in $f$ that is not in $h$ is either in $g^{\prime}$ or in $g^{\prime \prime}$.

Define for a subforest $g$ of a forest $f$

$$
q(g, f):=\prod_{j}\left(\prod_{v \in g} q_{1 j}^{p_{j}(v, g, f)} \cdot \prod_{v \in g^{c}} q_{2 j}^{p_{j}\left(v, g^{c}, f\right)}\right) .
$$

More intuitively, $q(g, f)$ counts for $v \in g$ the number of edges of colour $j$ in the path from $v$ to the root that have their lower vertex in $g^{c}$ and adds a factor $q_{1 j}$ for each of these, and $q(g, f)$ counts for $v \in g^{c}$ the number of edges of colour $j$ in the path 
from $v$ to the root that have their lower vertex in $g$ and adds a factor $q_{2 j}$ for each of these.

Theorem 4.3. Let $S\left(T_{n}\right)$ be the symmetric algebra on $n$-coloured trees as in Theorem 4.1.

(i) For a forest $f \in S\left(T_{n}\right)$ the coproduct defined by $\left(q_{11}, \ldots, q_{2 n}\right) \in k^{2 n}$ is given by the formula

$$
\Delta(f)=\sum_{g \subset f} q(g, f) g \otimes g^{c},
$$

where the sum is over all subforests $g$ of $f$.

(ii) The antipode of the Hopf algebra $S\left(T_{n}\right)$ with the coproduct of part (i) is given by

$$
S(f)=\sum_{k=1}^{|f|} \sum_{\cup_{i} g_{i}=f}(-1)^{k} g_{1} \cdots g_{k} \prod_{1 \leqslant j<k} q\left(g_{j}, g_{j} \cup \cdots \cup g_{k}\right),
$$

where we only sum over (ordered) partitions $f=g_{1} \cup \cdots \cup g_{k}$ of the forest $f$ with all forests $g_{i}$ non-empty.

Proof. We use induction with respect to the number of applications of $\lambda$ to show the first result. The formula is trivial for the empty tree. Let $t=\lambda\left(f_{1}, \ldots, f_{n}\right)$ be a tree and suppose (as the induction hypothesis) that the formula holds for all trees with less than $|t|$ vertices. Since $\Delta$ is an algebra morphism, it is clear the formula also holds for the forests $f_{i}$ since these consist of trees with less than $|t|$ vertices. Subforests of $t$ are either of the form $g=\cup_{i} g_{i}$, a (disjoint) union of subforests of the $f_{i}$, or of the form $g=r \cup\left(\cup_{i} g_{i}\right)$, a (disjoint) union of subforests of the $g_{i}$ together with the root. By definition and the induction hypothesis,

$$
\begin{aligned}
\Delta(t)= & \sum_{g_{i} \subset f_{i}} g_{1} \cdots g_{n} \otimes \lambda\left(g_{1}^{c}, \ldots, g_{n}^{c}\right) \cdot \prod_{i} q_{1 i}^{\left|g_{i}\right|} q\left(g_{i}, f_{i}\right) \\
& +\sum_{g_{i} \subset f_{i}} \lambda\left(g_{1}, \ldots, g_{n}\right) \otimes g_{1}^{c} \cdots \cdots g_{n}^{c} \cdot \prod_{i} q_{2 i}^{\left|g_{i}^{c}\right|} q\left(g_{i}, f_{i}\right) .
\end{aligned}
$$

But by Lemma 4.2 ,

$$
\prod q_{1 i}^{\left|g_{i}\right|} q\left(g_{i}, f_{i}\right)=\prod_{j}\left(\prod_{v \in g} q_{1 j}^{p_{j}(v, g, t)} \cdot \prod_{v \in g^{c}} q_{2 j}^{p_{j}\left(v, g^{c}, t\right)}\right)
$$

for $g=\cup_{i} g_{i}=g_{1} \cdots g_{n}$ and $g^{c}=r \cup\left(\cup_{i} g_{i}^{c}\right)=\lambda\left(g_{1}^{c}, \ldots, g_{n}^{c}\right)$; and

$$
\prod q_{2 i}^{\left|g_{i}^{c}\right|} q\left(g_{i}, f_{i}\right)=\prod_{j}\left(\prod_{v \in g} q_{1 j}^{p_{j}(v, g, t)} \cdot \prod_{v \in g^{c}} q_{2 j}^{p_{j}\left(v, g^{c}, t\right)}\right)
$$

for $g=r \cup\left(\cup_{i} g_{i}\right)=\lambda\left(g_{1}, \ldots, g_{n}\right)$ and $g^{c}=\cup_{i} g_{i}=g_{1}^{c} \cdots \cdot g_{n}^{c}$. Putting these together proves the formula for the coproduct.

To prove part (ii), consider for the moment an arbitrary graded connected bialgebra $A$. Write $\bar{A}=\bigoplus_{n \geqslant 1} A^{n}$ for its augmentation ideal. The antipode on $A$ applied 
to $x \in \bar{A}$ is given by

$$
S(x)=\sum_{k=0}^{\infty}(-1)^{k+1} \mu^{(k)} \circ \bar{\Delta}^{(k)}(x),
$$

where $\bar{\Delta}=\Delta-($ id $\otimes 1+1 \otimes$ id $)$, and $\mu^{(k)}: A^{\otimes k+1} \longrightarrow A$ and $\bar{\Delta}^{(k)}: A \longrightarrow A^{\otimes k+1}$ are defined using (co)associativity for $k>0$ and $\mu^{(0)}=\mathrm{id}=\bar{\Delta}^{(0)}$. (The sum in the formula for $S(x)$ is of course finite, and stops at $k$ for a homogeneous element $x$ of degree $k$.) For the special case of the bialgebra $S\left(T_{n}\right)$, part (i) of the theorem shows that the formula (4) for $S(x)$ specialises to the formula stated in part (ii). This proves the result.

Example 4.4. The Hopf algebra $C_{1}$ with the coproduct defined by $q_{11}=1$ and $q_{21}=0$ is the Connes-Kreimer Hopf algebra of rooted trees [3], [7], [10].

\section{Primitives of the dual}

Since $S\left(T_{n}\right)$ is commutative, we know by the Milnor-Moore Theorem [9] that the graded linear dual $S\left(T_{n}\right)^{*}$ is the universal enveloping algebra of the Lie algebra of its primitive elements. The result below provides an explicit formula for the Lie bracket on these primitive elements.

Corollary 5.1. Let $S\left(T_{n}\right)$ be the symmetric algebra on rooted trees with $n$-coloured edges, and let $\Delta$ be the coproduct defined by $\left(q_{11}, \ldots, q_{2 n}\right) \in k^{2 n}$ (cf. Theorem 4.3). The graded dual $S\left(T_{n}\right)^{*}$ is the universal enveloping algebra of the Lie algebra which as a vector space is spanned by elements $D_{t}$, where $t$ is a rooted tree in $S\left(T_{n}\right)$. The bracket is given by $\left[D_{s}, D_{t}\right]=D_{t} \bullet D_{s}-D_{s} \bullet D_{t}$, where

$$
D_{t} \bullet D_{s}=\sum_{x} \sum_{s \subset x, s^{c}=t} q(s, x) D_{x} .
$$

In this formula, the first sum ranges over all rooted trees $x$, and the second sum over subtrees of $x$ which are isomorphic to $s$ and whose complement $s^{c}$ is isomorphic to $t$.

Proof. For any cocommutative Hopf algebra we can define an operation $\bullet$ on the primitive elements, such that its commutator is the Lie bracket on primitive elements. Simply define $\bullet$ as the truncation of the product at degree $>1$, with respect to the primitive filtration $F$. In this case, $F_{m} C_{n}^{*}$ is spanned by the elements $D_{f}$ dual to forests $f$ consisting of at most $m$ trees. The product in $S\left(T_{n}\right)^{*}$ is determined by the coproduct in $S\left(T_{n}\right)$. For trees $s$ and $t$, and for a forest $f$, we can write the multiplication in $C_{n}^{*}$ as

$$
\left(D_{t} D_{s}\right)(f)=\left(D_{t} \otimes D_{s}\right) \Delta(f)
$$

thus, for a fixed $f$ we get a contribution $q(f, s) D_{f}$ for every subtree isomorphic to $s$ in $f$ with $t$ as complementary forest. The desired formula for $D_{t} \bullet D_{s}$ is obtained when we then restrict to the primitive part, i.e. the part where the forest $f$ is a tree (denoted $x$ in the formula). 
Recall (cf. Chapoton and Livernet [2]) that a pre-Lie algebra is vector space $L$ together with a bilinear operation $\bullet$ satisfying the identity

$$
(x \bullet y) \bullet z-x \bullet(y \bullet z)=(x \bullet z) \bullet y-x \bullet(z \bullet y) .
$$

The free pre-Lie algebra $L_{n}$ on $n$ generators is given by the vector space spanned by rooted trees with vertices labelled by elements of the set $\{1,2, \ldots, n\}$. The pre-Lie algebra product is given by grafting trees. For trees $s$ and $t$, and a vertex $v$ in $t$, denote by $t \circ_{v} s$ the tree obtained from $t$ and $s$ by attaching the root of $s$ to the vertex $v$ in $t$ by a new edge. Grafting preserves the labelling of the vertices. The pre-Lie algebra structure on $L_{n}$ is given by

$$
t \bullet s=\sum_{v \in t} t \circ_{v} s
$$

for trees $s$ and $t$.

Below, we denote by $\chi_{S}$ the characteristic function of a subset $S \subset X$ which has value 1 on $S$ and value 0 on $X-S$, and denote the vector space of primitive elements of a coalgebra $C$ by $P(C)$.

Theorem 5.2. Let $\mathbf{p} \subset\{1, \ldots, n\}$ and define $q_{1 j}=\chi_{\mathbf{p}}(j)$ and $q_{2 j}=0$ for $j=1, \ldots$, $n$. Consider the Hopf algebra structure on the symmetric algebra $S\left(T_{n}\right)$ on rooted trees with $n$-coloured edges that corresponds to this choice of $q_{i j}$.

(i) The product $D_{t} \bullet D_{s}=\sum_{x} \sum_{s \subset x, s^{c}=t} q(s, x) D_{x}$ of Corollary 5.1 defines a pre-Lie algebra structure on the vector space of primitive elements $P\left(C_{n}^{*}\right)$ of $S\left(T_{n}\right)^{*}$.

(ii) If $\mathbf{p}=\{1, \ldots, n\}$, then there is a natural inclusion of this pre-Lie algebra into the free pre-Lie algebra on $n$ generators. This inclusion identifies the vector space $P\left(C_{n}^{*}\right)$ with the subspace of the free pre-Lie algebra spanned by all sums $\sum_{i \in \mathbf{p}} t_{i}$, of trees with vertices coloured by $\mathbf{p}$ that only differ in that the colour of the root of $t_{i}$ is $i$.

Proof. Consider the general formula for $D_{t} \bullet D_{s}$ in Corollary 5.1. Note that for $q_{i j} \in\{0,1\}$ the coefficients $q(s, x)$ are either 0 or 1 . We can be more precise. Let $x$ and $t$ be trees. A product of subtrees $s=s_{1} \cdots s_{m} \subset x$ is $t$-admissible if $s^{c}$ contains the root of $x$ while $s$ is grafted onto $t=s^{c}$ by edges of colours $i_{1}, \ldots, i_{m} \in \mathbf{p}$ to vertices $v_{1}, \ldots, v_{m}$ respectively, each of which is connected to the root by edges having colours in $\mathbf{p}$. We only use this terminology for $m=1,2$. Note that $q(s, x)=0$ unless the corresponding subtree $s$ is $t$-admissible.

For trees $s, t$, and $u$, the pre-Lie identity follows from

$$
\left(D_{t} \bullet D_{s}\right) \bullet D_{u}-D_{t} \bullet\left(D_{s} \bullet D_{u}\right)=\sum_{x} \sum_{s \cdot u \subset x} D_{x},
$$

where the second sum is over $t$-admissible products $s \cdot u$. This proves part $(i)$ since the expression is symmetric in $s$ and $u$.

Before we prove $(i i)$ we study the pre-Lie algebra structure $\bullet$ in more detail. Let $m(t, s, x)$ be the number of $t$-admissible subtrees $s \subset x$. The operation $\bullet$ is then 
given by

$$
D_{t} \bullet D_{s}=\sum_{x} m(t, s, x) D_{x}
$$

where the sum is over all rooted trees. For our aims, it is better to use a different description of this pre-Lie algebra. We closely follow the strategy of Hoffman [6] in this respect. If $m(t, s, x) \neq 0$, it is exactly the order of the orbit of the root of the subtree $s$ under the action of the group Aut $(x)$ of automorphisms of $x$. If $s$ and $t$ are $n$-coloured trees and $v$ is a vertex in $t$, denote by $t{ }_{(v, i)} s$ the tree obtained from $t$ and $s$ by connecting the root of $s$ to the vertex $v$ by an edge of colour $i$. Let $n(t, s, x)$ be the number if vertices $v \in t$ such that $t \circ_{(v, i)} s=x$ for some $i \in \mathbf{p}$. Then for any such vertex $v$, the order of the orbit of $v$ in $t$ under the action of $\operatorname{Aut}(t)$ is exactly $n(t, s, x)$.

Define another pre-Lie algebra structure $\bullet^{\prime}$ on the same vector space $P\left(C_{n}^{*}\right)$, by

$$
D_{t} \bullet^{\prime} D_{s}=\sum_{x} n(t, s, x) D_{x}
$$

and denote this pre-Lie algebra by $P\left(C_{n}^{*}\right)^{\prime}$. For a subtree $s \subset x$, denote by $\operatorname{Aut}^{s}(x)$ the automorphisms of $x$ that pointwise fix $s$. Then, if $m(s, t, x) \neq 0$ we can write, following Hoffman [6],

$$
\begin{aligned}
m(t, s, x) & =\frac{|\operatorname{Aut}(x)|}{\left|\operatorname{Aut}^{s}(x)\right| \cdot|\operatorname{Aut}(t)|} \\
n(t, s, x) & =\frac{|\operatorname{Aut}(s)|}{\left|\operatorname{Aut}^{\{v\}}(t)\right|},
\end{aligned}
$$

for a vertex $v$ such that $t \circ_{(v, i)} s=x$ for some $i$. Since $\left|\operatorname{Aut}^{s}(x)\right|=\left|\operatorname{Aut}^{\{v\}}(t)\right|$ it follows that $D_{t} \longmapsto|\operatorname{Aut}(t)| D_{t}$ defines an isomorphism of pre-Lie algebras $P\left(C_{n}^{*}\right) \longrightarrow P\left(C_{n}^{*}\right)^{\prime}$ (in characteristic 0 ).

In the remainder of the proof, let $\mathbf{p}=\{1,2, \ldots, n\}$. We prove (ii) by constructing an inclusion $P\left(C_{n}^{*}\right)^{\prime} \longrightarrow L_{n}$. Note that

$$
D_{t} \bullet^{\prime} D_{s}=\sum_{v \in t} \sum_{i \in \mathbf{p}} D_{t \circ{ }_{(v, i)} s} .
$$

For an $n$-coloured tree $t$, denote by $\uparrow_{i}(t)$ the tree with coloured vertices obtained by moving the colour of each edge up to the vertex directly above it and colouring the root by $i$. Note that

$$
\uparrow_{j}\left(t \circ{ }_{(v, i)} s\right)=\uparrow_{j}(t) \circ_{v} \uparrow_{i}(s) .
$$

Let $\mathbf{p}=\{1, \ldots, n\}$ and consider $S\left(T_{n}\right)$ with the corresponding Hopf algebra structure as defined above. Define $\varphi: P\left(S\left(T_{n}\right)^{*}\right)^{\prime} \longrightarrow L_{n}$ from the pre-Lie algebra of primitives to the free pre-Lie algebra on $n$ generators by

$$
\varphi\left(D_{t}\right)=\sum_{j=1}^{n} \uparrow_{j}(t) .
$$


Then $\varphi$ is a linear embedding. Moreover, $\varphi$ preserves the pre-Lie algebra structure since

$$
\begin{aligned}
\varphi\left(D_{t} \bullet^{\prime} D_{s}\right) & =\sum_{(v, i)} \varphi\left(D_{t \circ_{(v, i)} s}\right) \\
& =\sum_{(v, i)} \sum_{j} \uparrow_{j}\left(t \circ_{(v, i)} s\right) \\
& =\sum_{v} \sum_{i, j} \uparrow_{j}(t) \circ_{v} \uparrow_{i}(s) \\
& =\varphi\left(D_{t}\right) \bullet \varphi\left(D_{s}\right) .
\end{aligned}
$$

Example 5.3. In the case of the Connes-Kreimer Hopf algebra (Example 4.4), Theorem 5.2 states that the dual Hopf algebra is the universal enveloping algebra of the free pre-Lie algebra on one generator. This was first proved by Chapton and Livernet [2].

\section{Infinitesimal coalgebra deformations}

In this section, we consider how the different Hopf algebra structures of Theorem 4.1 are related from the point of view of deformation theory. For simplicity we restrict our attention to the case $n=1$.

Let $A$ be a Hopf algebra. Recall (e.g., Gerstenhaber-Shack [5]) the bicomplex $C^{p q}(A)=\operatorname{Hom}\left(A^{\otimes p}, A^{\otimes q}\right)$ for $p, q \geqslant 1$. For $q$ fixed, the horizontal complex $C^{* q}$ is the Hochschild complex of the algebra $A$ with coefficients in the $A$ - $A$ bimodule $A^{\otimes q}$. For $p$ fixed, the vertical complex $C^{p *}$ is the Hochschild complex of the coalgebra $A$ with coefficients in the $A$ - $A$ bicomodule $A^{\otimes p}$.

Write $k[\delta]=k[x] /\left(x^{2}\right)$ for the ring of dual numbers, and $A[\delta]=A \otimes k[\delta]$.

An infinitesimal coalgebra deformation $\Delta_{\delta}$ of a Hopf algebra $A$ is a $k[\delta]$-linear map $\Delta_{\delta}: A[\delta] \longrightarrow A[\delta] \otimes_{k[\delta]} A[\delta]$ for which $\Delta_{\delta}$ makes $A[\delta]$ with the same multiplication and counit a Hopf algebra over $k[\delta]$, and such that evaluation at $\delta=0$ gives the original Hopf algebra structure on $A$.

The vector spaces $\operatorname{Der}\left(A, A^{\otimes q}\right)$ of algebra derivations form the kernel of the horizontal differential at the edge of the complex $C^{* q}(A)$, and thus a subcomplex of the coalgebra Hochschild complex. This complex starts out as

$$
\operatorname{Der}(A, A) \stackrel{d}{\longrightarrow} \operatorname{Der}\left(A, A^{\otimes 2}\right) \stackrel{d}{\longrightarrow} \operatorname{Der}\left(A, A^{\otimes 3}\right),
$$

where for $\varphi \in \operatorname{Der}(A, A)$ and $a \in A$,

$$
d \varphi(a)=\sum a^{\prime} \otimes \varphi\left(a^{\prime \prime}\right)-\Delta \varphi(a)+\sum \varphi\left(a^{\prime}\right) \otimes a^{\prime \prime},
$$

and for $\psi=\sum \psi^{\prime} \otimes \psi^{\prime \prime} \in \operatorname{Der}\left(A, A^{\otimes 2}\right)$,

$$
\begin{aligned}
d \psi(a)= & \sum a^{\prime} \otimes \psi\left(a^{\prime \prime}\right)-\sum \Delta \psi^{\prime}(a) \otimes \psi^{\prime \prime}(a) \\
& +\sum \psi^{\prime}(a) \otimes \Delta \psi^{\prime \prime}(a)-\sum \psi\left(a^{\prime}\right) \otimes a^{\prime \prime} .
\end{aligned}
$$


Infinitesimal coalgebra deformations of the Hopf $P$-algebra $A$ are in $1-1$ correspondence with classes in $H^{2}\left(\operatorname{Der}\left(A, A^{\otimes *}\right)\right)$.

Let us now turn to the example of the Hopf algebra $S\left(T_{1}\right)$ with the coproduct on trees given by $\Delta(s)=s \otimes 1+1 \otimes s$. (This is the coproduct induced by $\sigma_{1}=$ $\sigma_{2}=u \circ \varepsilon$, the composition of the unit and the counit.) If $q_{1}, q_{2} \in \delta \cdot k[\delta]$ and if we write $\lambda:=\lambda_{1}$, then the map $\Delta_{q_{1}, q_{2}}: S\left(T_{1}\right)[\delta] \longrightarrow S\left(T_{1}\right)[\delta] \otimes_{k[\delta]} S\left(T_{1}\right)[\delta]$ inductively defined by

$$
\Delta_{q_{1}, q_{2}}(\lambda(f))=\sum_{(f)} \lambda\left(f^{\prime}\right) \otimes q_{2}^{\left|f^{\prime \prime}\right|} f^{\prime \prime}+q_{1}^{\left|f^{\prime}\right|} f^{\prime} \otimes \lambda\left(f^{\prime \prime}\right)
$$

defines a coalgebra deformation of $S\left(T_{1}\right)$.

The Hopf algebra $S\left(T_{1}\right)$ is graded. Let us write $\operatorname{Der}_{0}\left(S\left(T_{1}\right), S\left(T_{1}\right)^{\otimes *}\right)$ for the subcomplex of $\operatorname{Der}\left(S\left(T_{1}\right), S\left(T_{1}\right)^{\otimes *}\right)$ consisting of those derivations that preserve the degree. Classes in $H^{2}\left(\operatorname{Der}_{0}\left(S\left(T_{1}\right), S\left(T_{1}\right)^{\otimes *}\right)\right)$ correspond to graded coalgebra deformations. The result below studies the deformations $\Delta_{q_{1}, q_{2}}$ as graded coalgebra deformations.

Proposition 6.1. Consider $S\left(T_{1}\right)$ with the coproduct induced by $\sigma_{1}=\sigma_{2}=u \circ \varepsilon$.

(i) The boundaries in $\operatorname{Der}_{0}\left(S\left(T_{1}\right), S\left(T_{1}\right)^{\otimes 2}\right)$ are the derivations $\varphi$ that can be written as

$$
\varphi(s)=\sum_{f} c_{s, f} \bar{\Delta}(f),
$$

for all trees $s$, and constants $c_{s, f} \in k$, and where the sum ranges over all forests $f$ such that $|s|=|f|$. As usual, $\bar{\Delta}=\Delta-(\mathrm{id} \otimes 1+1 \otimes \mathrm{id})$.

(ii) Let $q_{1} \equiv c_{1} \delta, q_{2} \equiv c_{2} \delta$, and $q_{1}^{\prime} \equiv d_{1} \delta, q_{2}^{\prime} \equiv d_{2} \delta$. Two graded infinitesimal coalgebra deformations $\Delta_{q_{1}, q_{2}}$ and $\Delta_{q_{1}^{\prime}, q_{2}^{\prime}}$ are equivalent iff $c_{1}-c_{2}=d_{1}-d_{2}$.

Proof. Let $\psi \in \operatorname{Der}_{0}\left(S\left(T_{1}\right), S\left(T_{1}\right)\right)$. Then $\psi(1)=0$, and $\psi$ is determined by its values on trees. Write $\psi$ in matrix form as $\psi(s)=\sum_{f} c_{s, f} f$, where the sum ranges over forests, and $c_{s, f} \in k$ are constants. Since we assume $\psi$ is graded, $c_{s, f}=0$ if $|f| \neq|s|$. Compute

$$
\begin{aligned}
d \psi(s) & =(\psi(s) \otimes 1+1 \otimes \psi(s))-\Delta(\psi(s)) \\
& =\sum_{f} c_{s, f}(f \otimes 1+1 \otimes f)-c_{s, f} \Delta(f) \\
& =-\sum_{f} c_{s, f} \bar{\Delta} f .
\end{aligned}
$$

For $\psi: S\left(T_{1}\right) \longrightarrow S\left(T_{1}\right)$ as above define the endomorphism $\Psi$ of $S\left(T_{1}\right)[\delta]$ by $\Psi(x)=$ $x+\psi(x) \delta$ for $x \in S\left(T_{1}\right)[\delta]$. Two infinitesimal coalgebra deformations $\Delta_{q_{1} q_{2}}$ and $\Delta_{q_{1}^{\prime} q_{2}^{\prime}}$ are equivalent iff we can find a derivation $\psi$ such that the corresponding map $\Psi$ satisfies, for all trees $s$,

$$
\Delta_{q_{1}^{\prime} q_{2}^{\prime}} \circ \Psi(s) \equiv \Psi \otimes \Psi \circ \Delta_{q_{1}, q_{2}}(s),
$$

or equivalently

$$
\Delta_{q_{1}^{\prime} q_{2}^{\prime}}(s+\psi(s) \delta) \equiv \Delta_{q_{1}, q_{2}}(s)+(\psi \otimes 1)\left(\Delta_{q_{1}, q_{2}}(s)\right) \delta+(1 \otimes \psi)\left(\Delta_{q_{1}, q_{2}}(s)\right) \delta .
$$


To compute this we only have to consider terms in $\Delta_{q_{1}^{\prime} q_{2}^{\prime}}(s)$ and $\Delta_{q_{1}, q_{2}}(s)$ corresponding to subforests $g \subset s$, such that

$$
\sum_{v \in g} p(v, g, s)+\sum_{v \in g^{c}} p\left(v, g^{c}, s\right) \leqslant 1,
$$

which means that either $g=s$, or $g^{c}=s$, or $s=g \circ_{r} \lambda(1)$, or $s=g^{c} \circ_{r} \lambda(1)$. We only need to consider terms in $\Delta_{q_{1}^{\prime} q_{2}^{\prime}}(\psi(s))$ corresponding to $g \subset s$, such that

$$
\sum_{v \in g} p(v, g, s)+\sum_{v \in g^{c}} p\left(v, g^{c}, s\right)=0,
$$

which means that either $g=s$, or $g^{c}=s$. From this it follows that $\Psi$ defines an equivalence of the two infinitesimal deformations iff the coefficients of $\delta$ match, which is to say

$$
\begin{aligned}
& \sum_{\left\{g \subset s \mid s=g \circ_{r} \lambda(1)\right\}} d_{1} g \otimes \lambda(1)+d_{2} \lambda(1) \otimes g+\sum_{f} c_{s, f} \Delta(f) \\
= & \sum_{\left\{g \subset s \mid s=g \circ_{r} \lambda(1)\right\}} c_{1} g \otimes \lambda(1)+c_{2} \lambda(1) \otimes g+\sum_{f} c_{s, f} f \otimes 1+c_{s, f} 1 \otimes f .
\end{aligned}
$$

Of course $c_{s, f} f \otimes 1+c_{s, f} 1 \otimes f$ is the primitive part of $\sum_{f} c_{s, f} \Delta(f)$. For the equality, we thus need $c_{s, f}=\left(c_{1}-d_{1}\right) m_{s g}=\left(c_{2}-d_{2}\right) m_{s g}$ if $f=\lambda(1) g$ for an $g$ such that $s=g \circ_{r} \lambda(1)$ and $m_{s g}$ is the cardinality of the orbit of the vertex in $s-g$ under the automorphism group of $s$. Choose the other $c_{s, f}$ equal to 0 .

\section{The general approach in the associative case}

The construction of bialgebras can be performed in much greater generality. Starting from an arbitrary Hopf operad $P$ one can construct the operad $P\left[\lambda_{n}\right]$ which has as algebras $P$-algebras together with an $n$-ary operation. Under conditions similar to those in Theorem 4.1 one can find a Hopf- $P$ algebra structure on the initial $P\left[\lambda_{n}\right]$-algebra (see $\left.[\mathbf{1 0}],[\mathbf{1 1}]\right)$. The explicit calculations for $P=\mathrm{Com}_{*}$, the operad for unital commutative algebras, is presented in the previous sections. These calculations can also be carried out for the operad $\mathrm{Ass}_{*}$ for unital associative algebras. In this section, we briefly state some of the resulting formulas.

Definition 7.1. Let $\mathcal{A}_{n}$ be the category which has as objects pairs $(A, \alpha)$, consisting of an associative unital algebra $A$ and a linear map $\alpha: A^{\otimes n} \longrightarrow A$, and which has as morphisms $\mathcal{A}_{n}((A, \alpha),(B, \beta))$ the algebra homomorphisms $f: A \longrightarrow B$ such that $\beta \circ f^{\otimes n}=f \circ \alpha$.

Let $\left(A_{n}, \lambda_{n}\right)$ be the initial object in the category $\mathcal{A}_{n}$. Then $A_{n}$ can be described as the free associative algebra on trees with edges coloured by $\{1, \ldots, n\}$ where in addition at each vertex the incoming edges with the same colour are endowed with a separate linear ordering. We also refer to these trees as planar $n$-trees. 
Theorem 7.2. The tensor algebra $A_{n}$ on planar $n$-trees has a natural family of graded connected Hopf algebra structures, indexed by sequences $\left(q_{11}, \ldots, q_{1 n}\right.$, $\left.q_{21}, \ldots, q_{2 n}\right) \in k^{2 n}$. The inductive description of the coproduct is given by the formula of Theorem 4.1 .

The $A_{n}$ together again form a simplicial algebra (cf. Remark 2.4).

Corollary 7.3. Let $A_{n}$ be the free associative algebra on the planar $n$-trees.

(i) The coproducts on $A_{n}$ of Corollary 7.3 are given by the closed formula

$$
\Delta(t)=\sum_{g \subset t} \prod_{j}\left(\prod_{v \in g} q_{1 j}^{p_{j}(v, g, t)} \cdot \prod_{v \in g^{c}} q_{2 j}^{p_{j}\left(v, g^{c}, t\right)}\right) g \otimes g^{c},
$$

where the product of trees is associative, non-commutative. The order of multiplication is given on the roots of the trees defined by the linear order on the incoming edges at each vertex and the partial order on the vertices.

(ii) The vector space of primitive elements of the dual is spanned by elements dual to planar $n$-trees. The Lie bracket is the commutator of the (nonassociative) product $\bullet$ given by

$$
D_{s} \bullet D_{t}=\sum_{x} \sum_{s \subset x, s^{c}=t} \prod_{j}\left(\prod_{v \in s} q_{1 j}^{p_{j}(v, s, x)} \cdot \prod_{v \in t} q_{2 j}^{p_{j}(v, t, x)}\right) D_{x}
$$

where $s, t$ and $x$ are trees with a linear ordering on the incoming edges of the same colour at each vertex and the inclusions of $s$ and $t$ in $x$ have to respect these orderings.

Proof. One can copy the proof of the commutative case almost verbatim. The only change is that we have to remember the ordering of up-going edges at each vertex.

Remark 7.4. Independently, Foissy [4] has found the formula for the Lie bracket in the case where $n=1, q_{11}=1$ and $q_{12}=0$ (and $A_{s s_{*}}$ is the underlying operad). He uses this formula to give an explicit isomorphism between the Hopf algebras $A_{1}$ and $A_{1}^{*}$ with this coproduct.

\section{Acknowledgements}

We are most grateful to the referee for his careful reading and detailed comments on an earlier version of this paper. During the period in which this article was written, PvdL was supported by Marie Curie Training Site Fellowships HPMT2000-00075 (Centre de Recerca Matemàtica, Barcelona) and HPMT-CT-2001-00075 (Université Paris Nord XIII). 
Homology, Homotopy and Applications, vol. 8(1), 2006

\section{References}

[1] C. Brouder and A. Frabetti, Renormalization of QED with planar binary trees. Euro. Phys. J. C 19 (2001), no. 4. pp. 715-741.

[2] F. Chapoton and M. Livernet, Pre-Lie algebras and the rooted trees operad. Int. Math. Res. Notices, 8 (2001), pp. 395-408. Also at arXiv:math.QA/0002069.

[3] A. Connes and D. Kreimer, Hopf algebras, renormalisation and noncommutative geometry. Commun. Math. Phys., 199 (1998), pp. 203-242.

[4] L. Foissy, Les algèbres de Hopf des arbres enracinés décorés. II. Bull. Sci. Math., 126 (2002), no. 4, pp. 249-288. Also at arXiv:math.QA/0105212.

[5] M. Gerstenhaber and S. D. Schack, Bialgebra cohomology, deformations, and quantum groups. Proc. Natl. Acad. Sci. USA, 87 (1990), no. 1, pp. 478-481.

[6] M. E. Hoffman, Combinatorics of rooted trees and Hopf algebras. Trans. Amer. Math. Soc., 355 (2003), no. 9, pp. 3795-3811 (electronic).

[7] D. Kreimer, On the Hopf algebra structure of perturbative quantum field theories. Adv. Theor. Math. Phys., 2 (1998), no. 2, pp. 303-334.

[8] J.-L. Loday and M. O. Ronco, Hopf algebra of the planar binary trees. Adv. Math., 139 (1998), no. 2, pp. 293-309.

[9] J. Milnor and J. Moore, On the structure of Hopf algebras. Ann. Math., 81 (1965), no. 2, pp. 211-264.

[10] I. Moerdijk, On the Connes-Kreimer construction of Hopf algebras. Cont. Math., 271 (2001), pp. 311-321. Also at arXiv:math-ph/9907010.

[11] P. P. I. van der Laan, Operads-Hopf algebras and coloured Koszul duality. Ph.D. thesis, Utrecht University (2004).

Pepijn van der Laan pepijnvanderlaan@planet.nl

Ieke Moerdijk moerdijk@math.uu.nl

Mathematical Institute

Utrecht University

Post Box 80010

3508TA Utrecht

The Netherlands

This article is available at http://intlpress.com/HHA/v8/n1/a8/ 